\title{
Gravitational hedgehog, stringy hedgehog and stringy structures
}

\author{
Özgür Delice*
}

March 1, 2019

\begin{abstract}
We investigate the solutions of Einstein equations such that an hedgehog solution is matched to different exterior or interior solutions via a spherical shell. In the case where both the exterior and the interior regions are hedgehog solutions or one of them is flat, then resulting spherical shell becomes a stringy shell. We also consider more general matchings and see that in this case the shell deviates from its stringy character and then discuss some other stringy structures.
\end{abstract}

\section{Introduction}

Topological defects may arise during phase transitions in the early Universe [1]. Depending on the type of the broken symmetry, monopoles, strings, domain walls and textures are produced [2. Among these, cosmic strings are studied extensively. The energy momentum tensor of an infinitely long straight cosmic string along $z$ axis is given by

$$
T_{z z}=-T_{00}=-\mu \delta(x) \delta(y)
$$

with other components vanishing [2]. The spacetime outside the straight cosmic string is flat but conical since the well known exterior metric of an infinitely long cosmic string contains an angular deficit. Namely in usual cylindrical coordinates:

$$
d s^{2}=-d t^{2}+d r^{2}+d z^{2}+(1-4 G \mu)^{2} r^{2} d \phi^{2}
$$

where the angular coordinate $\phi$ has the range $0 \leq \phi \leq 2 \pi$ and the $\mu$ is mass per unit length of the string.

Monopoles are formed when the vacuum manifold has surfaces which cannot be continuously shrunk to a point. If broken symmetry is a gauge symmetry then arising monopoles are like elementary particles. Their energy is mostly

\footnotetext{
*Bogazici University, Department of Physics, Bebek, Istanbul, Turkey; email:odelice@boun.edu.tr
} 
concentrated in a small region near the monopole core. However, if the symmetry is global, then global monopoles called hedgehogs arise. Their energy density is decreasing with the distance like $\sim r^{-2}$ thus their total energy diverges at large distances. In this paper we first review the gravitational field of such a hedgehog solution [4 arising from triplet of scalar fields. Then following [7, we see that the same gravitational field can also be constructed using an ensemble of straight radial cosmic strings intersecting at a central point. This solution is called the "string hedgehog" solution. We study possible matchings of this space-time to different interior or exterior space-times. Since the radial stress does not vanish, we cannot match this space-time smoothly. Thus, we will have an surface layer. We see that if we match this string hedgehog solution to another hedgehog solution or a flat solution, then the surface layer becomes a stringy shell. We will also determine under which conditions the shell, the exterior and interior regions satisfy some energy conditions. In the next section we make more general matchings. Then we discuss some other structures constructed by using cosmic strings.

\section{Gravitational field of a global monopole (Hedge- hog)}

The simplest model that gives rise to the hedgehog [4] is described by the Langrangian

$$
L=\frac{1}{2} \partial_{\mu} \phi^{a} \partial^{\mu} \phi^{a}-\frac{1}{4} \lambda\left(\phi^{a} \phi^{a}-\eta^{2}\right)^{2},
$$

where $\phi^{a}$ is a triplet of scalar fields, $a=1,2,3$. The model has a global $\mathrm{O}(3)$ symmetry, which is spontaneously broken to $\mathrm{U}(1)$. The field configuration describing a monopole is

$$
\phi^{a}=\eta f(r) x^{a} / r
$$

where $x^{a} x^{a}=r^{2}$. In flat space the hedgehog core has size $\delta \sim \lambda^{-1 / 2} \eta^{-1}$ and mass $M_{\text {core }} \sim \lambda^{-1 / 2} \eta$. Folloving [4] we can take $f(r)=1$ outside the core and the energy momentum tensor becomes

$$
T_{r r} \approx-T_{00} \approx-\eta^{2} / r^{2}, \quad T_{\theta \theta} \approx T_{\phi \phi} \approx 0 .
$$

Let us consider the general metric

$$
d s^{2}=-A^{2}(r) d t^{2}+B(r)^{2} d r^{2}+C(r)^{2} d \Omega_{2}^{2} .
$$

The nonzero components of the Einstein tensor for this metric are given by

$$
\begin{aligned}
G_{11} & =\frac{2 A_{r} C_{r}}{A B^{2} C}-\frac{1}{C^{2}}\left(1-\frac{C_{r}^{2}}{B^{2}},\right) \\
G_{22} & =G_{33}=\frac{A_{r r}}{A B^{2}}+\frac{C_{r r}}{B^{2} C}-\frac{A_{r} B_{r}}{A B^{3}}+\frac{A_{r} C_{r}}{A B^{2} C}-\frac{B_{r} C_{r}}{B^{3} C}, \\
G_{00} & =-\frac{2 C_{r r}}{B^{2} C}+\frac{2 B_{r} C_{r}}{B^{3} C}+\frac{1}{C^{2}}\left(1-\frac{C_{r}^{2}}{B^{2}}\right) .
\end{aligned}
$$


One immediately sees that choosing $A(r)=B(r)=$ const., $C(r)=\alpha r$ one gets the metric

$$
d s^{2}=-d t^{2}+d r^{2}+\alpha^{2} r^{2} d \Omega_{2}^{2}, \quad d \Omega_{2}=d \theta^{2}+r^{2} \theta d \phi^{2},
$$

with the only nonzero components of the Einstein tensor satisfying

$$
G_{00}=-G_{r r}=\frac{1-\alpha^{2}}{\alpha^{2} r^{2}} .
$$

This solution describes the asymptotic behavior of the exterior field of a global monopole outside the core and is first presented in 3 when studying spacetimes with angular deficit. Also, unlike straight cosmic strings, the exterior field of a gravitational monopole is not flat and it contains a solid deficit angle. by

The general solution of the metric (6) satisfying (5) with $C(r)=r$ is given

$$
A(r)=B^{-1}(r)=\left(1-8 \pi G \eta^{2}-\frac{2 G m}{r^{2}}\right) .
$$

Here $m$ is a constant of integration and $m \sim M_{\text {core }}$ [4]. It is shown in [5] and [6] that $m$ may be negative and is responsible for tiny repulsive force of the core of the hedgehog. Notice that for large and positive $m$, the solution corresponds to a hedgehog swallowed by a black hole. Actually, we can neglect the mass term $m$ for reasonable values of $\eta$ and $\lambda$ on the astrophysical scale [4]. When $m=0$, the general solution (11) reduces to (10) after rescaling $r$ and $t$ coordinates with $\alpha^{2}=\left(1-8 \pi G \eta^{2}\right)$.

Thus so far we have triplet of scalar fields $\phi^{a}$ of the form (44) generating gravitational field around the hedgehog (monopole). As shown in [7, the gravitational field around the hedgehog can also be constructed using an ensemble of radial cosmic strings.

\section{Gravitational field of a hedgehog string}

Let us suppose that we have an ensemble of radially oriented cosmic strings, whose gravitational field is given in (1), all of them intersecting at a central point. If we take the strength of each string to be very small but the number of strings very big, then the resulting configuration will approximately have spherical symmetry. In the continuum limit, this symmetry will be exact. This is called "string hedgehog" [7. The nonzero components of the energy-momentum tensor of this configuration will satisfy

$$
T_{r r}=-T_{00} .
$$

In this and the next section we will take $m=0$. Thus, we will use the metric (10). Note that the energy contained inside a sphere of radius $r_{0}$ is given by

$$
E=\int_{0}^{r_{0}} T_{00} e^{1} \wedge e^{2} \wedge e^{3}=4 \pi\left(1-\alpha^{2}\right) r_{0},
$$


where $e^{1}=d r, e^{2}=\alpha r d \theta, e^{3}=\alpha r \sin \theta d \phi$, and is linear in the proper radius of this sphere.

It is shown that we can have solutions with radial strings intersecting from a common point with [8, 9] or without [10, 11] a black hole at the center. It is also mentioned in those solutions that if mass per unit length of the strings (which is proportional to their angular deficit) is very small then we can go to the continuum limit. This actually corresponds to the situation we consider in this section.

\section{Stringy hedgeball}

In this section we match this string hedgehog solution to another hedgehog solution with the parameter $\beta$. When $\alpha=1$ or $\beta=1$, the interior or the exterior regions have flat Minkowski metric. Taking the exterior region Minkowskian may sound unphysical, but, if we only consider the limits where $m=0$ and $\alpha^{2} \approx$ $1 \quad\left(\eta^{2}<<1\right)$ than for large $r$ the exterior region becomes almost Minkowskian. Thus, we consider the solution with Minkowski exterior valid in these limits.

As we have emphasized before, for the string hedgehog solution, the energy density and the radial pressure $\rho_{r}$ is vanishing at $\infty$. In this section we will try to find out the answer of this question: Is it possible to match this spacetime to another string hedgehog solution with different parameter such that both the interior and the exterior regions and also the shell satisfy certain energy conditions? Since for exterior and interior regions we will have different radial pressures, from continuity, we cannot smoothly match these two regions. Thus, we need a surface layer (infinitely thin shell) at the boundary of these two regions.

We take the interior metric as (10) and the exterior metric as

$$
d s_{+}^{2}=-d t^{2}+\beta^{2} d \rho^{2}+\rho^{2} d \Omega_{2}^{2}
$$

There are several methods to calculate the energy momentum tensor of the shell [12-17]. Here we use an alternative method [17] which is equivalent to the standard method presented by Israel[12]. We also follow Lichnerowitz boundary conditions for surface layers [14]. The metrics interior and exterior to the hypersurface seperating these two regions must be continuous everywhere, but their derivatives with respect to the radial coordinate may contain discontinuities which give rise to energy momentum tensor of the shell. This is the condition to have a surface layer at $r=r_{0}$. To do this we choose $\rho=\rho(r)$ and using the boundary conditions given below we can write both metrics continuous at $r=r_{0}$. From continuity conditions at $r=r_{0}$ we have:

$$
\rho\left(r_{0}\right)=\frac{\alpha}{\beta} r_{0} ; \quad \rho_{, r}\left(r_{0}\right)=1 .
$$

Choosing $\rho(r)=a r+b$ and using boundary conditions one gets

$$
\rho(r)=r+(\alpha / \beta-1) r_{0} .
$$


Thus the exterior and interior metrics become

$$
\begin{aligned}
& d s_{+}^{2}=-d t^{2}+d r^{2}+\beta^{2}\left(r+(\alpha / \beta-1) r_{0}\right)^{2} d \Omega_{2}^{2} \\
& d s_{-}^{2}=-d t^{2}+d r^{2}+\alpha^{2} r^{2} d \Omega_{2}^{2} .
\end{aligned}
$$

Or with the help of the Heaviside step function we can combine both interior and exterior metrics in the form

$$
d s^{2}=\theta\left(r-r_{0}\right) d s_{+}^{2}+\theta\left(r_{0}-r\right) d s_{-}^{2} .
$$

After calculating the Einstein tensor for this metric, the terms proportional to Dirac delta function will give the energy momentum tensor of the shell and the terms proportional to the step functions give the interior and exterior solutions. Thus, the energy-momentum tensor for the whole spacetime can be expressed as

$$
T_{\mu \nu}=T_{\mu \nu}^{(-)} \theta\left(r_{0}-r\right)+T_{\mu \nu}^{(+)} \theta\left(r-r_{0}\right)+T_{\mu \nu}^{(0)} \delta\left(r-r_{0}\right),
$$

where

$$
T_{\mu \nu}^{(k)}=\operatorname{diag}\left(\rho^{(k)}, p_{i}^{(k)}\right), \quad k= \pm, 0 ; \quad i=r, \theta, \phi .
$$

Calculating the Einstein tensor, one gets for the nonzero components

$$
\begin{aligned}
& \rho^{(-)}=-p_{r}{ }^{(-)}=\frac{1-\alpha^{2}}{\alpha^{2} r^{2}}, \quad \rho^{(+)}=-p_{r}^{(+)}=\frac{1-\beta^{2}}{\beta^{2} r^{2}}, \\
& \rho^{(0)}=\frac{2(\alpha-\beta)}{\alpha r}, \quad p_{\theta}^{(0)}=p_{\phi}^{(0)}=\frac{(\beta-\alpha)}{\alpha r} .
\end{aligned}
$$

So, we have matched a string hedgehog space-time to another string hedgehog solution via a surface layer at the boundary. Since we do not want to discuss the cosmological solutions where $\alpha^{2}=1-8 \pi G \eta^{2}$ is negative, we limit the range of the parameters $\alpha$ and $\beta$ as $-1 \leq\{\alpha, \beta\} \leq 1$ since we have $0<\left\{\alpha^{2}, \beta^{2}\right\} \leq 1$.

For an energy momentum tensor of the form (17) we have the weak energy condition $\left(\rho \geq 0, p_{i} \geq 0\right)$, the dominant energy condition $\left(\rho \geq\left|p_{i}\right|\right)$ and strong energy condition $\left(\rho+\sum_{i} p_{i} \geq 0\right)$. Thus, the weak energy condition is not satisfied since $p_{i}$ is negative. When $\alpha$ and $\beta$ have the same signs, to have a positive energy density, we need $|\alpha|>|\beta|$. When they have different signs the energy density is always positive. The shell has the equation of state:

$$
\rho=-\left(p_{\theta}+p_{\phi}\right) .
$$

Then, when the shell has positive energy density, it satisfies dominant and strong energy conditions. Note that both interior and exterior regions also satisfy these energy conditions for these ranges of the parameters. The total energy of the shell is $E=8 \pi \alpha(\alpha-\beta) r_{0}$.

We can interpret this shell as a shell composed of uniformly distributed cosmic strings lying on the surface of a sphere. We call a surface as stringy in the energy momentum tensor of such shell satisfies $\sum_{i} p_{i}=-\rho$. 
Thus when we want to embed a stringy hedgehog spacetime to another hedgehog solution, a stringy spherical shell arises. For a certain range of the parameters, both shell and string hedgehog spacetime satisfies energy conditions.

If we take $\beta=1$ the exterior region becomes flat Minkowski spacetime. In this case to have a shell satisfying energy conditions, we need $-1<\alpha<0$. We can call this solution with flat exterior a "stringy hedgeball" solution. Or, we can discuss the opposite situation. If we take $\alpha=1$, then the interior region of the spherical shell becomes flat. For this case, to satisfy energy conditions, one needs $0<\beta<1$.

\section{Generalization}

In this section we consider the interior and the exterior metrics $d s_{ \pm}^{2}$ as hedgehogSchwarzschild-de Sitter solutions with different coordinates for interior and exterior regions such as $(t, r, \theta, \phi)$ and $(\tau, R, \theta, \phi)$ for the metric of the form (6) where the metric functions are given as

$$
\begin{array}{r}
A_{-}(r)=B_{-}^{-1}(r)=\left(\alpha^{2}-\frac{2 m}{r}-\frac{\Lambda_{-}}{3} r^{2}\right)^{1 / 2} \quad C_{-}(r)=r \\
A_{+}(R)=B_{+}^{-1}(R)=\left(\beta^{2}-\frac{2 M}{R}-\frac{\Lambda_{+}}{3} R^{2}\right)^{1 / 2} \quad C_{+}(R)=R .
\end{array}
$$

We require them to be continuous at $r=r_{0}$. We again keep the interior metric as it is and for the exterior metric we choose $R=R(r)=a r+b$ and $\tau=E t$. Then we have the following boundary conditions:

$$
R\left(r_{0}\right)=r_{0}, \quad R_{, r}\left(r_{0}\right)=\frac{A_{+}\left(r_{0}\right)}{A_{-}\left(r_{0}\right)}, \quad E=\frac{A_{-}\left(r_{0}\right)}{A_{+}\left(r_{0}\right)} .
$$

Our exterior metric becomes

$$
d s_{+}^{2}=-A_{+}^{2}(r) d t^{2}+A_{+}^{-2}(r) d t^{2}+R^{2}(r) d \Omega_{2}^{2} .
$$

with

$$
\begin{aligned}
A_{+}(r) & =\sqrt{\beta^{2}-\frac{2 M}{R(r)}-\frac{\Lambda_{+} R^{2}}{3}} \frac{A_{-}\left(r_{0}\right)}{A_{+}\left(r_{0}\right)}, \\
R(r) & =\frac{A_{+}\left(r_{0}\right)}{A_{-}\left(r_{0}\right)} r+\left(1-\frac{A_{+}\left(r_{0}\right)}{A_{-}\left(r_{0}\right)}\right) r_{0} .
\end{aligned}
$$

The energy momentum tensor of the shell has the following components

$$
\rho^{(0)}=2 K\left(r_{0}\right), \quad p_{\theta}^{(0)}=p_{\phi}^{(0)}=-K\left(r_{0}\right)+L\left(r_{0}\right),
$$

where

$$
\begin{aligned}
K\left(r_{0}\right) & =\frac{A_{-}\left(r_{0}\right)}{r_{0}}\left(A_{-}\left(r_{0}\right)-A_{+}\left(r_{0}\right)\right), \\
L\left(r_{0}\right) & =-\frac{A_{-}\left(r_{0}\right)}{A_{+}\left(r_{0}\right)}\left(\frac{\Lambda_{+} r_{0}}{3}-\frac{M}{r_{0}^{2}}\right)+\left(\frac{\Lambda_{-} r_{0}}{3}-\frac{m}{r_{0}^{2}}\right) .
\end{aligned}
$$


As pointed out in [7] we can superpose the Schwarzschild-de Sitter solution with the hedgehog solution. We do this in this section and we match this spacetime to same exterior solution with different parameters. This solution is quite general since choosing some parameters zero we can recover the more simple solutions such as when $m, M, \Lambda_{ \pm}$vanishing, the solution reduces to the stringy shell solution we have presented in the previous section. If $\Lambda_{ \pm},=0, \alpha=\beta=1$ we have a shell around a black hole 19 .

Note that this shell no longer satisfies (20). However, whenever the parameters other than $\alpha, \beta$ go to zero, $L\left(r_{0}\right)$ also goes to zero and we recover (20). The presence and the difference of these parameters deviates shell from its stringy character. For this general case we can specify an equation of state $p=p(\rho)$ and solve for parameters. We can have even be a domain wall satisfying $p_{\theta}^{(0)}=p_{\phi}^{(0)}=-\rho^{(0)}$ if the parameters satisfy $K\left(r_{0}\right)+L\left(r_{0}\right)=0$ or a shell of photons satisfying $T_{\mu}^{\mu(0)}=0$ if the relation $4 K\left(r_{0}\right)-L\left(r_{0}\right)=0$ holds.

\section{Some structures arising from cosmic strings}

Here, we will discuss very incomplete list of solutions of Einstein equations giving rise to cosmic stringy structures. By cosmic stringy structures we mean a $d$ dimensional hypersurface in 4 dimensional space-time $(d \leq 3)$ such that on this hypersurface the nontrivial components of the Energy-Momentum tensor satisfy

$$
\sum_{i=1}^{d} p_{i}=-\rho
$$

The famous solution of Vilenkin [20] describing an infinitely long straight cosmic string is our first example of this structures where strings taken as a $\delta$-functional source $(d=1)$. On the string the condition (30) is satisfied.

One example of the two dimensional string structure satisfying the condition (30) is the stringy hollow cylinder solution where the infinitely long cylinder is constructed from straight strings [21]. In this solution, the interior region is flat and the exterior region is given by locally flat cosmic string metric (2). This solution is extended to the rotating hollow stringy cylinder case [22]. The solution we have presented in section (4) is another example of cosmic stringy surfaces where the strings lie on the surface of a sphere. Unlike the hollow cylinder case, either both or the interior or the exterior regions are not vacuum but contains radial strings.

The solutions with multiple parallel or nonparallel straight cosmic strings moving with different velocities are given in [25]. Thus using this solution, we can even orient strings non parallel. Using a different method, Arik et. al. presented a solution [20] where a straight cosmic string decay into conical surfaces at its end points and ending up as a stringy sheet with radial strings. This solution is extended to the other cases 24] including a string changing into a cylinder, a cylinder changing into a cylinder with different radius and a 
cylinder decaying into conical surfaces at its endpoints and ending up as a sheet with radial strings.

The Gott-Hischok-Linet interior solutions [26]- 28] are three dimensional examples of the cosmic stringy structures where they consider a solid cylinder filled with long string fibers with $p_{z}=-\rho$. The first two solutions considered the energy density is constant in the cylinder whereas the third one considered a variable energy density. Both solutions matched to the string exterior smoothly. These are the first examples of stringy volumes.

The coasting universe [30 is a cosmological solution where the universe is dominated by cosmic strings [29]. The equation of state of the fluid fills up this universe is $p=-1 / 3 \rho$ which can be thought of made with the straight strings.

Recently, another example of stringy volumes and surfaces is given 31. In toroidal coordinates, they give a solution with an interior solution with stringlike equation of state, a surface layer with again a string-like equation of state and a locally AdS exterior.

Unlike straight strings, curved strings have nonzero gravitational potential [2]. Hence, they cannot be static. It is known that a loop of string may oscillate or, due to its large tension, either emits radiation and vanishes completely, or collapses and emits some portion of its energy as radiation and forms a black hole [33. To have a loop stable one needs to support if again collapse. A circular string solution is given in 32 as a initial value problem. The solution we presented in section 4 presents strings lying on a surface of a sphere. In our solution these strings are supported by radial strings.

Actually, there could be more solutions that we are not aware of. However, the solutions we have mentioned reflects the richness of the situation of constructing more complex structures such as stringy surfaces or volumes using cosmic strings.

\section{Conclusion}

In this paper using thin shell formalism, we have glued some interior solutions in spherical coordinates to an exterior solution via a shell where interior and exterior solutions have the same character with different parameters.

After reviewing the global hedgehog (monopole) and the string hedgehog solutions, we have first chosen the interior and the exterior regions as the string hedgehog solution which can be made up with an ensemble of radial cosmic strings and we have calculated the energy momentum tensor of the shell seperating these two regions. We see that this infinitely thin shell is composed of cosmic strings lying on the surface of the sphere. For certain ranges of the parameters, the interior, the exterior and the shell satisfy the dominant energy condition.

Then we have chosen the interior and the exterior regions as HedgehogSchwarzschild-de Sitter solutions with different parameters, and we have seen that for this case the shell is no longer stringy. The shell in this case can be a domain wall or a photonic shell. 
We have also reviewed some of the structures constructed from cosmic strings. We have seen that using cosmic strings one can construct more complex structures such as stringy surfaces or volumes. We also showed that one can orient them on a surface of a sphere. There could be other structures awaiting their exploration.

\section{Acknowlegment}

I would like to thank M. Arık for reading the manuscript and useful discussions.

\section{References}

[1] Kibble T W B 1976 J. Phys. A 91387

[2] Vilenkin A and Shellard E P S 1994 Cosmic Strings and other Topological Defects (Cambridge: Cambridge University Press).

[3] Sokolov D D and Starobinsky A A 1977 Dokl. Akad. Nauk. SSSR 2341043 [1977 Sov. Phys. Dokl. 22 312]

[4] Bariola M and Vilenkin A 1989 Phys. Rev. Lett. 63341

[5] Harari D and Lousto C 1990 Phys. Rev. D 422626

[6] Shi X and Li X 1991 Class. Quantum Grav. 8761

[7] Guendelman E I and Rabinowitz A 1991 Phys. Rev. D 443152

[8] Aryal M, Ford L H and Vilenkin A 1986 Phys. Rev. D 342263

[9] Ivanov M G, 2002 Grav. Cosmol. 8171

[10] Dowker J S and Chang P 1992 Phys. Rev. D 463458

[11] Frolov V P, Fursaev D V and Page D N 2002 Phys. Rev. D 65104029

[12] Israel W 1966 Nuovo Cimento B44, 1; Israel W 1967 ibid. 48 463(E)

[13] Darmois G, Mémorial des Sciences Mathematiques (Paris: GauthierVillars), Fasc 25.

[14] Lichnerowicz A, Théories Relativistes de la Gravitation et de Électromagnétisme (Paris: Masson) p 61.

[15] Papapetreu A and Hamoi A 1968 Ann. Inst. Henri. Poicaré 9179

[16] Taub A H 1980 J Math. Phys. 211423

[17] Mansouri R and Khorrami M 1996 J. Math. Phys. 375672 
[18] Hawking S W and Ellis G F R 1972 The Large Scale Structure of SpaceTime(Cambridge University Press) ch 4.4

[19] Frauendiener J, Hoenselaers C and Konrad W 1990 Class. Quantum Grav. 7585

[20] Vilenkin A 1981 Phys. Rev. D 23852

[21] Tsoubelis D 1989 Class. Quantum Grav. 6101

[22] Clément G and Zouzou I 1994 Phys. Rev. D 507271

[23] Arik E, Arik M, Kornfilt J, Saygili K and Yildiz A 1995 Phys. Lett. B 352 224

[24] Delice O 2000 Ms. Thesis Bogazici University

[25] Letelier P S and Gal'tsov D V 1993 Class. Quantum Grav. 10 L101

[26] Gott J R 1985 Astrophys. J. 288422

[27] Hiscock W A 1985 Phys. Rev. D 323288

[28] B. Linet 1985 Gen Relativ. Gravit. 171109

[29] Vilenkin A 1984 Phys. Rev. Lett. 531016

[30] Kolb E W 1989 Astrophy. J 344543

[31] Krisch J P and Glass E N 2003 J. Math. Phys.44 3046

[32] Frolov V P, Israel W and Unruh W G 1989 Phys. Rev. D 391084

[33] Hawking S W 1990 Phys. Lett. B 24636 